\title{
Glueball solitons
}

\author{
M. Baker \\ University of Washington, Seattle, Washington 98195 \\ J. S. Ball \\ University of Utah, Salt Lake City, Utah 84112 \\ F. Z. Chen \\ University of Washington, Seattle, Washington 98195 \\ F. Zachariasen \\ California Institute of Technology, Pasadena, California 91125
}

(Received 7 May 1985)

\begin{abstract}
From the effective action of long-range Yang-Mills theory, we derive a classical spherical solution which can be interpreted as a glueball. The field configurations in the gauge we have used describe a system with one unit of color-electric flux crossing any median plane, and thus can be regarded as two gluons bound together. The glueball has $J^{P}=0^{+}$. Its mass depends on the parameters in a phenomenological potential, and lies in the range of one to four $\mathrm{GeV}$ for all choices of those parameters which we have considered.
\end{abstract}

\section{INTRODUCTION}

This paper describes the application of an effective Lagrangian for long-range Yang-Mills theory, ${ }^{1}$ recently proposed by us, to the study of classical glueballs. The Lagrangian is the simplest one satisfying the following conditions: (i) that in the Abelian limit it reduces to one describing a dielectric medium with dielectric constant $\epsilon=\square / M^{2}$; (ii) that it is expressed in terms of the electric vector potential $C_{\mu}$ rather than the usual magnetic vector potential, as is appropriate for a medium satisfying (i) ${ }^{2}$ (iii) that it is invariant under magnetic gauge transformations $C_{\mu} \rightarrow U^{-1} C_{\mu} U-i / g U^{-1} \partial_{\mu} U$.

The Lagrangian is

$$
\begin{gathered}
L_{\text {eff }}=\frac{1}{4} 2 \operatorname{Tr}\left[2 G_{\mu \nu} \widetilde{F}_{\mu \nu}+\widetilde{F}_{\mu \nu} \frac{\mathscr{D}^{2}(C)}{M^{2}} \widetilde{F}_{\mu \nu}\right. \\
\left.-\frac{1}{f^{2}} G_{\mu \nu} G_{\mu \nu}-W(\widetilde{F})\right] .
\end{gathered}
$$

Here

$$
\begin{aligned}
& G_{\mu \nu}=\partial_{\mu} C_{\nu}-\partial_{\nu} C_{\mu}-i g\left[C_{\mu}, C_{\nu}\right], \\
& \mathscr{D}_{\mu}(C)=\partial_{\mu}-i g\left[C_{\mu},\right],
\end{aligned}
$$

and $g$ is related to the usual Yang-Mills coupling constant $e$ via $e g=4 \pi$. The function $W$ is a phenomenological "potential," which we discuss below. In (1.1), the independent fields are $C_{\mu}$ and $\widetilde{F}_{\mu v} ; \widetilde{F}_{\mu \nu}$ is related to the dual electromagnetic field tensor and necessarily appears as an independent variable. Its use replaces nonlocal operators such as $1 / \mathscr{D}^{2}(C)$. The antisymmetric field $G_{\mu v}$ defines the electric displacement and magnetic $H$ field:

$$
G_{i j}=\epsilon_{i j k} D_{k}
$$

and

$$
G_{0 i}=H_{i}
$$

We refer to the analogous components of $\widetilde{F}_{\mu \nu}$ as the electric and magnetic fields,

$$
\widetilde{F}_{i j}=-\epsilon_{i j k} E_{k}
$$

and

$$
\widetilde{F}_{0 i}=-B_{i},
$$

though these are the actual electric and magnetic fields only in the case that all fields are Abelian-that is, all fields are in a single color direction.

We wish to obtain a spherically symmetric solution to the classical field equations obtained from (1.1). This, as will become clear, is to be interpreted as a glueball. In I we studied cylindrically symmetric solutions to (1.1). We found that there exist solutions which are vortexlike tubes of quantized color-electric flux, so that (1.1) is the Lagrangian for a medium which is like a dual superconductor. $^{2,3}$ It therefore confines color charge, just as an ordinary superconductor would confine magnetic monopoles. These cylindrical solutions arise when $W$ is such as to cause dynamical symmetry breaking, through its having a minimum at a nonzero magnetic field $\mathbf{B}$, much like what occurs via the Higgs mechanism. The nonperturbative vacuum here is, however, oriented in space according to the direction of the flux tube. The true vacuum is an average over all orientations of this direction, and gaugeinvariant quantities are the same in both the oriented and true vacuums. We shall see that very much the same situ- 
ation obtains in the spherical case. In spite of the nonzero $B$ field far from either flux tube or glueball, physically relevant quantities, such as the energy density, vanish exponentially at long range.

\section{DERIVATION OF THE EFFECTIVE LAGRANGIAN}

Let us first discuss the extent to which the effective Lagrangian (1.1) can be shown to be a consequence of YangMills theory.

The Lagrangian of the Yang-Mills quantum theory is of course known: $L(A)=-\frac{1}{4} F_{\mu \nu} F_{\mu \nu}$, with

$$
F_{\mu v}=\partial_{\mu} A_{v}-\partial_{v} A_{\mu}-i e\left[A_{\mu}, A_{v}\right] \text {. }
$$

This is equal to a Lagrangian $L(C)$, expressed in terms of the electric vector potential $C_{\mu}$, but what explicit function $L(C)$ is of $C$ is not known, because we do not in general know how to translate from $A$ to $C$. What is known ${ }^{4}$ is that $L(C)$ is invariant under dual gauge transformations

$$
C_{\mu} \rightarrow U^{-1} C_{\mu} U-\frac{i}{g} U^{-1} \partial_{\mu} U
$$

just as $L(A)$ is invariant under ordinary gauge transformations

$$
A_{\mu} \rightarrow V^{-1} A_{\mu} V-\frac{i}{e} V^{-1} \partial_{\mu} V,
$$

with $e g=4 \pi$. Therefore the dual proper vertex functions, associated with $L(C)$, satisfy Ward identities of the same form as do the ordinary proper vertices, coming from $L(A)$.

We seek to construct the simplest effective Lagrangian $L_{\text {eff }}(C)$ corresponding to $L(C)$ which contains the essential long-range features of the theory. The most important feature to be maintained is current conservation. In many problems, both classical and quantum mechanical, the requirement of current conservation has major effects at long range.

One example of this is provided by the motion of an excitation through the quantum ground state of liquid helium. ${ }^{5}$ If one attempts to form a localized excitation, and improves the trial wave function sufficiently to guarantee current conservation, then the necessary backflow in the surrounding medium induces effects at long range falling off only as a dipole.

The same feature appears in classical incompressible fluid flow. An exact solution to Euler's equation and the equation of continuity (current conservation) is Hill's spherical vortex, ${ }^{6}$ which describes a vortex propagating in a fluid originally at rest. Again the flow at long range is that of a dipole; no localized excitation exists.

These examples indicate the importance of preserving exact current conservation in obtaining long-distance physical properties. This is particularly true for YangMills theory where perturbation theory yields longdistance singularities in the gluon propagator $\Delta_{A}(x, y)=\left\langle A_{\mu}(x) A(y)\right\rangle$.

The Fourier transform of $\Delta_{A}(q)$ is given in terms of the dielectric constant $\epsilon\left(q^{2}\right)$ by

$$
\Delta_{A}\left(q^{2}\right)=-\frac{1}{q^{2} \epsilon\left(q^{2}\right)} .
$$

The dielectric constant $\epsilon\left(q^{2}\right) \equiv \Gamma_{2}^{A}(q)$ in turn is determined in terms of $\Delta_{A}$ and the vertex functions $\Gamma_{3}^{A}$ and $\Gamma_{4}^{A}$ by the Schwinger-Dyson equations. Corresponding equations determine higher vertex functions $\Gamma_{n}^{A}$ in terms of $\Gamma_{n+1}^{A}$ and $\Gamma_{n+2}^{A}$.

Current conservation is guaranteed if the vertex functions satisfy the Ward identities. Combining the Ward identities with the Schwinger-Dyson equations therefore yields a sequence of iterations, at each stage maintaining current conservation, determining the vertices. In the first step of this sequence the Ward identity for $\Gamma_{3}^{A}$ is used to (approximately) express $\Gamma_{3}^{A}$ in terms of $\Delta_{A}$. The Dyson equation for $\epsilon$ then becomes an integral equation for $\epsilon$, containing the backflow (the global rearrangement of the vacuum current distribution necessary to preserve current conservation).

We have solved this integral equation and found the following long-distance behavior: ${ }^{7,8}$ as $q^{2} \rightarrow 0$,

$$
\begin{aligned}
& \epsilon\left(q^{2}\right) \rightarrow-\frac{q^{2}}{M^{2}}, \\
& \Delta_{A}\left(q^{2}\right) \rightarrow \frac{M^{2}}{q^{4}} .
\end{aligned}
$$

The next step in the approximation procedure should now be to use the Ward identity for $\Gamma_{4}^{A}$ to express it in terms of $\Gamma_{3}^{A}$, and then to solve the coupled Dyson equations for $\epsilon=\Gamma_{2}^{A}$ and $\Gamma_{3}^{A}$. But, because of the singular long-distance behavior for $\Delta_{A}\left(q^{2}\right)$ which we found at the first stage, the procedure is evidently not convergent. Each successive stage gives a more singular $\Delta_{A}$; indeed, the exact propagator may well not exist. ${ }^{9}$

These singularities make it clear that the vector potentials $A_{\mu}$ are not convenient variables in terms of which to describe long-distance Yang-Mills theory. We need a more natural set of variables. What these are is suggested by (2.2) and (2.3).

In the first stage of our iteration procedure, we found that all vertices $\Gamma_{n}^{A}$ vanished as the momenta go to zero. Therefore, at this stage, long-distance Yang-Mills theory is an Abelian theory with a propagator $\Delta_{A}\left(q^{2}\right)=M^{2} / q^{4}$. But an Abelian theory can be equally well described in terms of the electric vector potentials $C_{\mu}$ and for these the propagator is

$$
\Delta_{C}\left(q^{2}\right)=-\frac{1}{q^{2} \mu\left(q^{2}\right)},
$$

where the permeability $\mu$ is related to the dielectric constant by $\epsilon \mu=1$. Since the dielectric constant vanishes at long range, the permeability is singular: as we see from (2.2), as $q^{2} \rightarrow 0$

$$
\mu\left(q^{2}\right) \rightarrow-\frac{M^{2}}{q^{2}}+\frac{1}{f^{2}}
$$

(we have added a constant, called $1 / f^{2}$, to represent the next term in the low $-q^{2}$ expansion of $\mu$; on physical grounds, $f^{2}$ must be positive to have a positive energy density, exponential decay and the right sign in the dielectric constant) and so from (2.4) we find that 


$$
\Delta_{C}\left(q^{2}\right) \rightarrow-\frac{f^{2}}{q^{2}-f^{2} M^{2}}
$$

as $q^{2} \rightarrow 0$. In terms of the $C_{\mu}$ variables, therefore, our approximate current conserving solution of long-range Yang-Mills theory is well behaved. The singularity at $q^{2}=0$ is not present; instead the $C_{\mu}$ propagator develops a mass. The backflow due to current conservation screens magnetic charge, and (2.6) describes a magnetic superconductor.

At the Abelian level, since (2.3) and (2.6) describe exactly the same physics, the use of the electric vector potentials shows us that our current conserving approximation to long-range Yang-Mills theory is not in fact singular. The singular behavior of $\Delta_{A}\left(q^{2}\right)$ does not imply any physical singularity; it only reflects the fact that the variables $A_{\mu}(x)$ are singular at long range.

We can now use the $C_{\mu}$ to extend our discussion beyond the Abelian limit. For higher order, corrections to a massive theory will not, in general, produce new lowmomentum singularities. Therefore we expect the exact $\Delta_{C}$ to also be finite as $q^{2} \rightarrow 0$. Furthermore, we know that the exact Yang-Mills theory is invariant under magnetic gauge transformations, ${ }^{4}$ and consequently that the exact vertices $\Gamma_{n}^{c}$ are current conserving and satisfy Ward identities. Hence the exact effective Lagrangian, expressed in terms of $C_{\mu}$, must satisfy the following conditions: (i) that in the Abelian limit the permeability reduces to (2.5), and (ii) that it is invariant under the gauge transformations

$$
C_{\mu} \rightarrow U^{-1} C_{\mu} U-\frac{i}{g} U^{-1} \partial_{\mu} U
$$

The minimal Lagrangian satisfying these constraints is

$$
L_{\mathrm{eff}}(C)=\frac{1}{4} G_{\mu v}\left(\frac{M^{2}}{\mathscr{D}^{2}(C)}-\frac{1}{f^{2}}\right) G_{\mu v},
$$

where

$$
G_{\mu \nu}=\partial_{\mu} C_{\nu}-\partial_{\nu} C_{\mu}-i g\left[C_{\mu}, C_{\nu}\right]
$$

and

$$
\mathscr{D}_{\mu}(C)=\partial_{\mu}-i g\left[C_{\mu},\right]
$$

Equation (2.7) is (essentially) our effective Lagrangian (1.1). It is important to emphasize that, while the Abelian limit of (2.7) coincides with (2.3), once the non-Abelian parts are included there is no simple relation between $C$ and $A$, or between $\Delta_{C}\left(q^{2}\right)$ and $\Delta_{A}\left(q^{2}\right): L_{\text {eff }}(C)$ does not describe a theory for which $\Delta_{A}\left(q^{2}\right) \rightarrow M^{2} / q^{4}$ as $q^{2} \rightarrow 0$.

It is convenient to rewrite (2.7) in terms of the dual field tensor $\widetilde{F}_{\mu \nu}$ in the form

$L_{\mathrm{eff}}(C)=\frac{1}{2} G_{\mu \nu} \widetilde{F}_{\mu \nu}-\frac{1}{4} \widetilde{F}_{\mu \nu} \frac{\mathscr{D}^{2}(C)}{M^{2}} \widetilde{F}_{\mu \nu}-\frac{1}{4 f^{2}} G_{\mu \nu} G_{\mu \nu}$,

with $\widetilde{F}$ and $C$ as independent variables. This avoids the use of the operator $1 / \mathscr{D}^{2}(C)$.

Equation (2.10) is the minimal choice for $L_{\text {eff }}$ meeting our constraints. Additional gauge-invariant terms involving $C_{\mu}$ could be present, but these will vanish exponentially at large distances. However there could be additional gauge-invariant terms depending on $\widetilde{F}_{\mu \nu}$ because, as we will see later, $\widetilde{F}_{\mu \nu}$ becomes constant at large distances. We therefore add a phenomenological "potential" $W(\widetilde{F})$ to (2.10), thus finally arriving at our complete effective Lagrangian (1.1).

Physically $W(\widetilde{F})$ plays the role of the Higgs potential $V(\phi)$. Spontaneous symmetry breaking can occur when $W$ has a minimum at a nonzero value of $\widetilde{F}$. The existence of quantized electric flux tubes, and therefore of electric confinement, and (as we shall see) of classical glueballs all follow from a nontrivial minimum of $W$. The basic field configurations, geometrically and in color, are also fixed. Qualitatively, further details about $W$ are irrelevant. But quantitatively, numerical values for various quantities of physical interest, such as the string tension, the vacuum energy, and the glueball mass, depend at least somewhat on the details of $W$.

We do not know $W$. It represents that part of our effective Lagrangian which we do not know how to derive from the quantum Yang-Mills theory, and appears because the Abelian limit and gauge invariance are not sufficient to uniquely fix the effective long-range Lagrangian.

The simplest thing to do is to make $W$ a polynomial, in analogy to the standard choice of the Higgs potential: $V(\phi)=\mu^{2}|\phi|^{2}+\lambda|\phi|^{4}$. We have, however, imposed a constraint on $W$, namely, that it should vanish when $\widetilde{F}$ is Abelian-that is, when all its components lie in a single color direction. This is in order that in the Abelian limit, our effective Lagrangian reduces to one describing a dielectric medium with dielectric constant and permeability $\mu=1 / \epsilon=M^{2} / \square$. This constraint means that $W$ contains no term of order $\widetilde{F}^{2}$, so we take $W$ to be of order $\widetilde{F}^{4}$ and $\widetilde{F}^{6}$.

The only possible fourth-order forms are $\operatorname{Tr}\left[\widetilde{F}_{\mu v}, \widetilde{F}_{\alpha \beta}\right]^{2}$ and $\operatorname{Tr}\left[\widetilde{F}_{\mu \nu}, \widetilde{F}_{\nu \alpha}\right]^{2}$. There are, however, many different sixth-order terms. In the cylindrical problem described in I, only one fourth-order and two sixth-order forms are independent; for example, $\operatorname{Tr}\left(\left[\widetilde{F}_{\mu \nu}\left[\widetilde{F}_{\alpha \beta}, \widetilde{F}_{\lambda \sigma}\right]\right]\right)^{2}$ and $\left(\operatorname{Tr} \widetilde{F}_{\mu \nu}\left[\widetilde{F}_{\nu \lambda}, \widetilde{F}_{\lambda \mu}\right]\right)^{2}$. In the spherical problem, described here, there are more. We write, as one convenient choice,

$$
\begin{aligned}
W= & \lambda_{1} 2 \operatorname{Tr}\left[\widetilde{F}_{\mu \nu} \widetilde{F}_{\alpha \beta}\right]^{2}+\lambda_{2} 2 \operatorname{Tr}\left[\widetilde{F}_{\alpha \beta},\left[\widetilde{F}_{\mu \nu}, \widetilde{F}_{\sigma \lambda}\right]\right]^{2}+\lambda_{3}\left(2 \operatorname{Tr} \widetilde{F}_{\mu \nu}\left[\widetilde{F}_{\nu \alpha}, \widetilde{F}_{\alpha \mu}\right]\right)^{2}+\lambda_{4} 2 \operatorname{Tr}\left[\widetilde{F}_{\mu \nu}\left[\widetilde{F}_{\mu \nu}, \widetilde{F}_{\sigma \lambda}\right]\right]^{2} \\
& +\lambda_{5} 2 \operatorname{Tr}\left[\widetilde{F}_{\mu \nu}\left[\widetilde{F}_{\sigma \alpha}, \widetilde{F}_{\alpha \lambda}\right]\right]\left[\widetilde{F}_{\mu \nu}\left[\widetilde{F}_{\sigma \beta}, \widetilde{F}_{\beta \lambda}\right]\right]+\lambda_{6} 2 \operatorname{Tr}\left[\widetilde{F}_{\mu \nu}\left[\widetilde{F}_{\nu \lambda}, \widetilde{F}_{\lambda \sigma}\right]\right]\left[\widetilde{F}_{\alpha \beta},\left[\widetilde{F}_{\beta \gamma}, F_{\gamma \mu}\right]\right]
\end{aligned}
$$


The parameters here are constrained by requiring that the asymptotic value of $W$ for our spherical solution is the same as it was in the flux-tube problem, so that the vacuum energy densities are the same in both cases.

\section{DERIVATION OF THE SPHERICAL SOLUTION}

We begin our discussion by writing down the field equations, in covariant form, following from the effective Lagrangian (1.1); they are

$\mathscr{D}_{\mu}(C) G_{\mu \nu}-\mathscr{D}_{\mu}(C) \widetilde{F}_{\mu \nu}-\frac{i}{2}\left[\mathscr{D}_{\nu}(C) \widetilde{F}_{\alpha \beta}, \widetilde{F}_{\alpha \beta}\right]=0$

and

$$
\mathscr{D}^{2}(C) \widetilde{F}_{\mu \nu}+G_{\mu \nu}-\frac{\partial W}{\partial \widetilde{F}_{\mu \nu}}=0 .
$$

Here we have adopted the same scaling used in I, to eliminate explicit coupling constants and masses from the field equations.

The most general spherically symmetric form for the independent fields $C_{0}, \mathrm{C}, \mathrm{E}$, and $\mathrm{B}$ is ${ }^{10}$

$$
\begin{aligned}
C_{0}= & C_{0}(r) \hat{\mathbf{x}} \cdot \mathbf{T} \\
\mathbf{C}= & -\frac{1+\phi_{2}(r)}{r} \hat{\mathbf{x}} \times \mathbf{T}-\frac{\phi_{1}(r)}{r} \hat{\mathbf{x}} \times(\hat{\mathbf{x}} \times \mathbf{T}) \\
& +A_{1}(r) \hat{\mathbf{x}}(\hat{\mathbf{x}} \cdot \mathbf{T}) \\
\mathbf{E}= & E_{1}(r) \hat{\mathbf{x}} \times \mathbf{T}-E_{2}(r) \hat{\mathbf{x}} \times(\hat{\mathbf{x}} \times \mathbf{T})+E_{3}(r) \hat{\mathbf{x}}(\hat{\mathbf{x}} \cdot \mathbf{T})
\end{aligned}
$$

$$
\mathbf{B}=B_{1}(r) \hat{\mathbf{x}} \times \mathbf{T}-B_{2}(r) \hat{\mathbf{x}} \times(\hat{\mathbf{x}} \times \mathbf{T})+B_{3}(r) \hat{\mathbf{x}}(\hat{\mathbf{x}} \cdot \mathbf{T}) .
$$

In these equations $\hat{\mathbf{x}}$ is the unit radius vector and $r$ is the spherical radial coordinate. The generator of $\mathrm{SU}(2)$ color $T$ is normalized to $\left[T_{a}, T_{b}\right]=i \epsilon_{a b c} T_{c}$. We are interested in static solutions so that all functions in (3.3) are independent of time.

From the potentials we can calculate the color-electric displacement

$$
\begin{aligned}
\mathbf{D}= & -\frac{1}{r}\left(\frac{\partial \phi_{1}}{\partial r}+A_{1} \phi_{2}\right) \widehat{\mathbf{x}} \times \mathbf{T} \\
& +\frac{1}{r}\left(\frac{\partial \phi_{2}}{\partial r}-A_{1} \phi_{1}\right) \widehat{\mathbf{x}} \times(\hat{\mathbf{x}} \times \mathbf{T}) \\
& -\frac{1}{r^{2}}\left(1-\phi_{1}{ }^{2}-\phi_{2}{ }^{2}\right) \widehat{\mathbf{x}}(\hat{\mathbf{x}} \cdot \mathbf{T})
\end{aligned}
$$

and the color magnetic field

$$
\begin{aligned}
\mathbf{H}= & \frac{C_{0} \phi_{1}}{r} \hat{\mathbf{x}} \times \mathbf{T}-\frac{C_{0} \phi_{2}}{r} \hat{\mathbf{x}} \times(\hat{\mathbf{x}} \times \mathbf{T}) \\
& -\frac{\partial C_{0}}{\partial r} \hat{\mathbf{x}}(\hat{\mathbf{x}} \cdot \mathbf{T}) .
\end{aligned}
$$

Under the gauge transformation

$$
U=\exp [i \alpha(r) \hat{\mathbf{x}} \cdot \mathbf{T}]
$$

the fields transform as follows: $C_{0}, E_{3}$, and $B_{3}$ are invariant,

$$
\begin{aligned}
& \phi_{1} \rightarrow \phi_{1} \cos \alpha-\phi_{2} \sin \alpha, \\
& \phi_{2} \rightarrow \phi_{1} \sin \alpha+\phi_{2} \cos \alpha,
\end{aligned}
$$

with the same transformation for the pairs $E_{1}$ and $E_{2}$, and $B_{1}$ and $B_{2}$, and finally

$$
A_{1} \rightarrow A_{1}+\partial \alpha / \partial r
$$

At large distances from the localized spherical excitation we want the solution to approach the vacuum up to a gauge transformation. Therefore, asymptotically

$$
\begin{aligned}
& \mathrm{D}=\mathbf{H}=0, \\
& \mathscr{D}_{i} E_{j}=\mathscr{D}_{i} B_{j}=0, \\
& \frac{\partial W}{\partial E_{i}}=\frac{\partial W}{\partial B_{i}}=0 .
\end{aligned}
$$

Consequently, using Eqs. (3.4) we must have, asymptotically,

$$
C_{0}(r)=0
$$

and

$$
\phi_{1}(r)+i \phi_{2}(r)=\exp \left[i \int^{r} d r^{\prime} A_{1}\left(r^{\prime}\right)\right] .
$$

Similarly, Eq. (3.7b) implies that asymptotically

$$
\begin{aligned}
\frac{\partial B_{1}}{\partial r}+A_{1} B_{2} & =\frac{\partial B_{2}}{\partial r}-A_{1} B_{1} \\
& =\frac{\partial B_{3}}{\partial r}=B_{1}+\phi_{1} B_{3}=B_{2}+\phi_{2} B_{3}=0,
\end{aligned}
$$

together with similar conditions when $B$ is replaced by $E$. Therefore $B_{3}$ and $E_{3}$ are constants asymptotically, which we call $-b$ and $-e$, respectively, determined from (3.7c), and furthermore

$$
\begin{aligned}
& B_{1}+i B_{2}=b\left(\phi_{1}+i \phi_{2}\right), \\
& E_{1}+i E_{2}=e\left(\phi_{1}+i \phi_{2}\right) .
\end{aligned}
$$

We are, of course, interested in solutions which are not simply gauge transformations of the vacuum. Suppose we look for a solution in the gauge in which $A_{1}=0$. In this gauge, the asymptotic behavior is seen from (3.8) and (3.10) to be

$$
\begin{aligned}
& C_{0} \rightarrow 0, \\
& \phi_{1} \rightarrow \cos \beta, \phi_{2} \rightarrow \sin \beta, \\
& E_{1} \rightarrow e \cos \beta, \quad E_{2} \rightarrow e \sin \beta, \\
& B_{1} \rightarrow b \cos \beta, \quad B_{2} \rightarrow b \sin \beta,
\end{aligned}
$$

where $\beta$ is a constant.

The vacuum solution is $C_{0}=\mathbf{C}=0, \quad \mathbf{E}=-e \mathbf{T}$, $\mathbf{B}=-b \mathrm{~T}$ everywhere; from Eq. (3.3) this corresponds to (3.11) with $\beta=-\pi / 2$. We may gauge transform the vac- 
uum solution, using (3.5) with $\alpha(r)=\pi \tanh r$, to obtain a solution with asymptotic behavior

$$
\begin{aligned}
& C_{0} \rightarrow 0, \\
& \mathbf{C} \rightarrow-\frac{2}{r} \widehat{\mathbf{x}} \times \mathbf{T}, \\
& \mathbf{E} \rightarrow e(\mathbf{T}-2 \widehat{\mathbf{x}} \hat{\mathbf{x}} \cdot \mathbf{T}), \\
& \mathbf{B} \rightarrow b(\mathbf{T}-2 \widehat{\mathbf{x}} \hat{\mathbf{x}} \cdot \mathbf{T}) ;
\end{aligned}
$$

however it also has $A_{1}=\pi \operatorname{sech}^{2} r \neq 0$. Let us therefore look for a solution in the $A_{1}=0$ gauge, but with asymptotic behavior given by (3.12); this will then not be gauge equivalent to the vacuum. As we will see below, we can in fact find such a solution, with $\phi_{1}, E_{1}$, and $B_{1}$ as well as $A_{1}$, vanishing everywhere.

Before we explicitly exhibit this solution, let us use its asymptotic behavior, (3.12), to discuss the color-electric flux associated with it.

In $\mathrm{SU}(\boldsymbol{N})$ Yang-Mills theory the gauge-invariant electric flux through a loop $L$ is given by the Mandelstam ${ }^{4}$ 't Hooft ${ }^{11}$ magnetic Wilson loop through the equation

$$
P\left[\exp \left[i g \oint_{L} \mathbf{C} \cdot d l\right]\right] \equiv e^{(2 \pi i / N) \Phi},
$$

where $\mathrm{C}=\sum_{\alpha} T_{\alpha} \mathrm{C}^{\alpha}$ and the $T^{\alpha}$ are the $\mathrm{SU}(N)$ generators in the fundamental representation. In (3.13) $P$ is the usual path-ordering operator. Equation (3.13) determines the electric flux $\Phi$ modulo $N$ in units of the quark charge $e / N$ where $e$ is the Yang-Mills coupling constant.

In I we obtained the solution for an $n=1 \mathrm{SU}(2)$ electric flux tube in the gauge where $\mathbf{C}$ was along a fixed direction
$\left(T_{3}\right)$ in color space, so for this case the path ordering is irrelevant, and we found $g \oint_{L} \mathrm{C} \cdot d l=2 \pi T_{3}$ for any loop enclosing the flux tube. Thus the flux was $\Phi=1$ in units of $e / 2$. For this case, since $\mathbf{C}$ was Abelian, the gaugeinvariant flux coincides with the (in general gaugevariant) ordinary definition of flux $\Phi=\int_{s} \mathbf{D} \cdot d \mathbf{S}$ across a surface $S$ enclosed by $L$.

In the spherical case, however, the gauge invariant flux defined through (3.13) vanishes, since we can choose a gauge in which $\mathbf{C} \rightarrow 0$ asymptotically. The ordinary flux, crossing any plane passing through the origin with normal $\hat{\mathbf{n}}$, however, is found from

$$
g \int \mathbf{D} \cdot d \mathbf{S}=g \oint \mathbf{C} \cdot d l=-(1+\sin \beta)(2 \pi \hat{\mathbf{n}} \cdot \mathbf{T})
$$

as can be seen from (3.11), and can therefore take on any value between 0 and -2 , depending on the gauge choice.

The vanishing of the gauge-invariant flux in the spherical case reflects the fact that, in contrast with the flux tube, the glueball is not topologically stable and can decay. But this does not mean that there are no singularity free static solutions; in fact the glueball solution we exhibit below is just such a nontopological soliton.

Our solution is obtained in the gauge where $\beta=\pi / 2$, so in this gauge the conventional gauge-dependent flux is two, in units of $e / 2$. That is, two units of $e / 2$ color flux cross any plane through the origin. In this gauge, therefore, the solution corresponds to a gluon [of charge $2(e / 2)$ ] above the plane and an antigluon [charge $-2(e / 2)$ ] below, so that one can visualize the glueball as a two-gluon bound state.

The field equations (3.1) and (3.2) with the choice $A_{1}=\phi_{1}=E_{1}=B_{1}=0$, and with $C_{1} \equiv-\left(1+\phi_{2}\right) / r$, become

$$
\begin{aligned}
& \widetilde{\nabla}^{2} C_{0}-2\left(C_{1}{ }^{2}-E_{2}{ }^{2}+B_{2}{ }^{2}\right) C_{0}-\frac{4 C_{0} C_{1}}{r}+\frac{\partial B_{3}}{\partial r}+\frac{2\left(B_{3}-B_{2}\right)}{r}-2 C_{1} B_{2}=0, \\
& \widetilde{\nabla}^{2} C_{1}-\left(C_{1}{ }^{2}-C_{0}{ }^{2}-E_{2}{ }^{2}-E_{3}{ }^{2}+B_{2}{ }^{2}+B_{3}{ }^{2}\right) C_{1}-\frac{3 C_{1}{ }^{2}}{r}+\frac{\left(E_{2}-E_{3}\right)^{2}}{r} \\
& \nabla^{2} E_{2}-\left(C_{1}{ }^{2}-C_{0}{ }^{2}\right) E_{2}-\frac{2\left(E_{2}-E_{3}\right)}{r^{2}}-\frac{2 C_{1}\left(E_{2}-B_{3}\right)^{2}}{r}-\frac{\partial E_{2}}{\partial r}-\frac{E_{2}-E_{3}}{r}+C_{1} E_{3}+\frac{C_{0}{ }^{2}}{r}+C_{0} B_{2}=0, \\
& \nabla^{2} E_{3}-2 C_{1}{ }^{2} E_{3}-\frac{4\left(E_{3}-E_{2}\right)}{r}-\frac{4 C_{1}\left(E_{3}-E_{2}\right)}{r}-\frac{2 C_{1}}{r}-C_{1}{ }^{2}=-\frac{\partial W}{\partial E_{3}}, \\
& r^{2}
\end{aligned}
$$


In these expressions we use the notation

$$
\nabla^{2}=\frac{1}{r^{2}} \frac{\partial}{\partial r} r^{2} \frac{\partial}{\partial r}
$$

and

$$
\widetilde{\nabla}^{2}=\frac{\partial}{\partial r} \frac{1}{r^{2}} \frac{\partial}{\partial r} r^{2}
$$

Equations (3.14) comprise a system of six coupled nonlinear second-order differential equations in one variable.

Asymptotically we wish to choose the "potential" $W$ so that the derivatives $\partial W / \partial E_{2}$, etc., on the right-hand side of Eqs. (3.14c)-(3.14f) vanish. That is, asymptotically we expect to approach the (oriented) vacuum, which is a minimum of $\boldsymbol{W}$. The oriented vacuum can have a nonvanishing value of $\widetilde{F}_{\mu v}$, since the excitation for which we are looking can pick out a preferred direction in color space, and in real space. The oriented vacuum cannot, therefore, be the same as the true vacuum, since in the true vacuum $\widetilde{F}_{\mu \nu}$ must vanish. Nevertheless, as discussed in $I$, the value of any gauge-invariant quantity, such as the gluon condensate $G_{2}$, will be the same in the oriented and in the true vacuum.

If the asymptotic solution has a nonzero value of $\widetilde{F}_{\mu \nu}$, then we have spontaneous symmetry breaking. In the cylindrical case described in $I$, we found such a solution, with a nonvanishing asymptotic value of $\mathbf{B}$, and this solution had a lower-energy density than the trivial perturbative vacuum, in which all fields vanish. We therefore look for a similar situation here.

As we have indicated, we search for a solution with asymptotic behavior (3.12). A nonzero asymptotic $\mathbf{E}$ is, as (3.12) indicates, allowed by the field equations. However, again as in the cylindrical case, a nonzero $\mathbf{E}$ inhibits the existence of exponentially falling fields at infinity, as can be seen from the sign of the $E^{2}$ terms in (3.14a) and (3.14b). Furthermore, on physical grounds we want the oriented vacuum here to be like that in the flux tube, which, we recall, was purely magnetic. Therefore we shall limit ourselves to solutions with $\mathbf{E} \rightarrow 0$, and our oriented vacuum is characterized by $\mathbf{B} \rightarrow b(\mathbf{T}-2 \widehat{\mathbf{x}} \mathbf{\mathbf { x }} \cdot \mathbf{T})$ as $r \rightarrow \infty$, as is evident from (3.12).

The rest of the solution now proceeds in a way similar to I. We first determine the leading long-distance corrections to the asymptotic form by writing

$$
\begin{aligned}
& C_{0}(r)=c_{0}(r), \\
& C_{1}(r)=-\frac{2}{r}+c_{1}(r), \\
& E_{2}(r)=e_{2}(r), \\
& E_{3}(r)=e_{3}(r), \\
& B_{2}(r)=b+b_{2}(r), \\
& B_{3}(r)=-b+b_{3}(r),
\end{aligned}
$$

and linearizing the field equations in $c_{0}, c_{1}$, etc. These linear equations are solvable analytically, and we find that $c_{0}$ and $c_{1}$ are proportional to the spherical Bessel function $k_{1}(a r), e_{2}+e_{3}$ and $b_{2}+b_{3}$ are proportional to $k_{0}(a r)$, and $2 e_{2}-e_{3}$ and $2 b_{2}-b_{3}$ are proportional to $k_{2}(a r)$. The number $\alpha$ is determined by an eigenvalue equation in terms of the parameters appearing in the "potential" $W$. For appropriate ranges of those parameters, there are six solutions for $\alpha$ of this equation with positive real part, and therefore there are six independent exponentially falling solutions for the functions $c_{0}(r), c_{1}(r)$, etc.

As $r \rightarrow 0$, the field equations also admit six independent nonsingular solutions, for which $C_{0}$ and $C_{1}$ vanish linearly with $r$ while the other four functions approach constants.
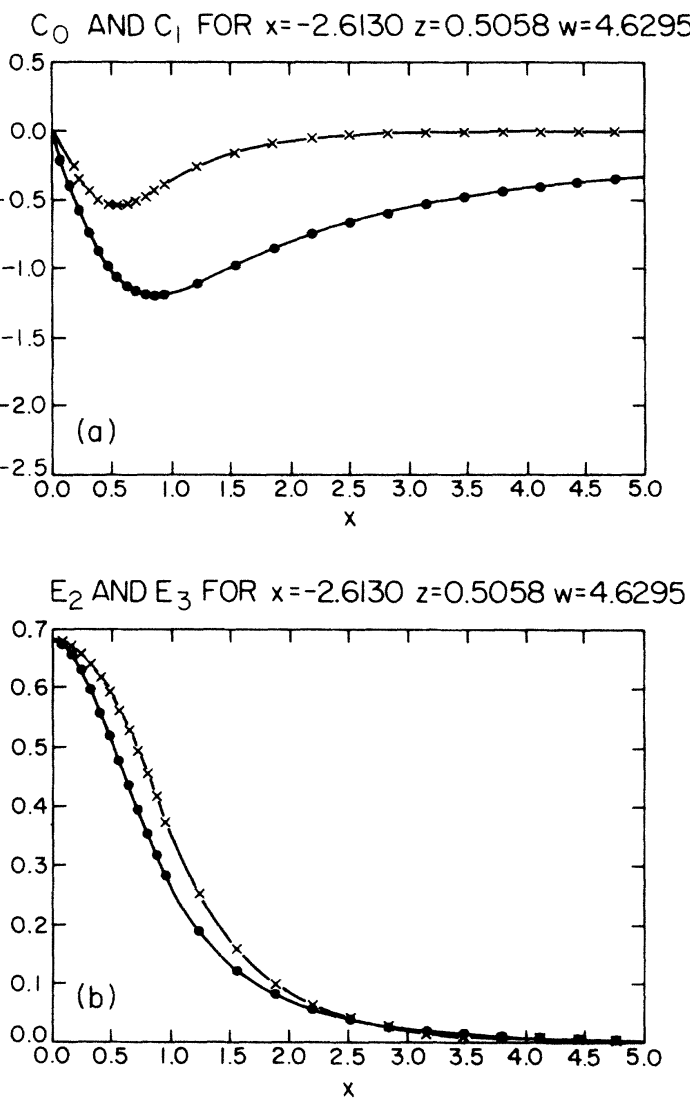

$B_{2}$ AND B 3 FOR $x=-2.6130 z=0.5058 w=4.6295$

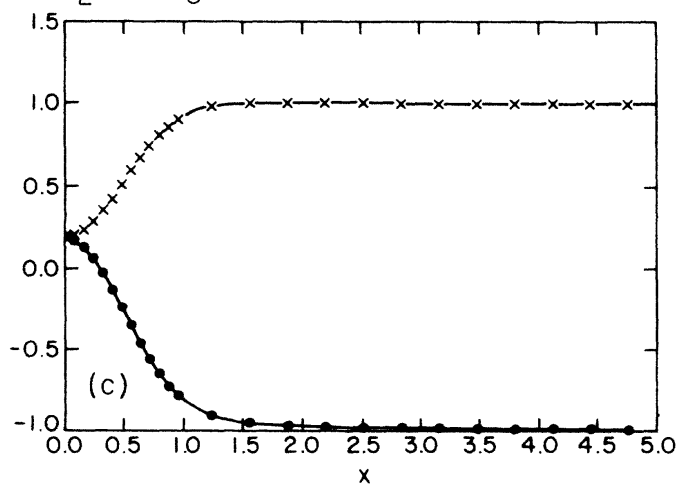

FIG. 1. (a) Plots of $C_{0}(r)$ and $C_{1}(r)$ vs $r$. The dots are $C_{1}$ and the crosses are $C_{0}$. (b) Plots of $E_{2}(r)$ (dots) and $E_{3}(r)$ (crosses) vs $r$. (c) Plots of $B_{2}(r)$ (dots) and $B_{3}(r)$ (crosses) vs $r$. 
The solution is now obtained numerically by integrating the finite solution at infinity, with six parameters, inward and the finite solution at the origin, also with six parameters, outward, and then matching all twelve parameters at some intermediate point.

In Figs. 1(a)-1(c) we plot the six functions $C_{0}(r)$, etc., as functions of the radius $r$ which, as in $I$, is measured in units of the mass scale $M_{f}=f M$ in terms of the parameters in the original effective Lagrangian. The figures are drawn for a typical choice of the parameters in $W$.

In Fig. 2 we plot the energy density (times $r^{2}$ ) as a function of radius. It, of course, falls off exponentially as it should. The mass of the spherical excitation-the glueball mass-is the integral of this energy density:

$$
M_{\text {glueball }}=E_{\text {total }}=\int_{0}^{\infty} 4 \pi r^{2} d r \mathscr{H}(r),
$$

where the Hamiltonian density $\mathscr{H}$ is
$H$ FOR $x=-2.6130 z=0.5058 w=4.6295$

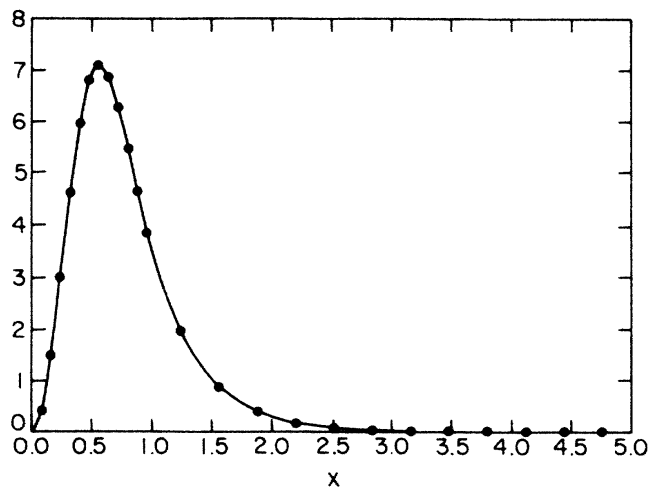

FIG. 2. The energy density as a function of radius. What is plotted is $r^{2} \mathscr{H}(r)$.

$$
\begin{aligned}
\mathscr{H}(r)= & {\left[\frac{\partial C_{1}}{\partial r}+\frac{C_{1}}{r}\right)^{2}+\frac{1}{2}\left(\frac{\partial C_{0}}{\partial r}\right)^{2}+\frac{1}{2}\left(\frac{2 C_{1}}{r}+C_{1}{ }^{2}\right)^{2}+\left(\frac{C_{0}}{r}+C_{0} C_{1}\right)^{2}+C_{0}{ }^{2} B_{2}{ }^{2}-3 C_{0}{ }^{2} E_{2}{ }^{2} } \\
& +\left(\frac{\partial E_{2}}{\partial r}\right)^{2}+\frac{1}{2}\left[\frac{\partial E_{3}}{\partial r}\right)^{2}+\left(E_{2}-E_{3}\right)^{2}\left[\frac{2}{r^{2}}+\frac{2 C_{1}}{r}\right)+C_{1}{ }^{2}\left(E_{2}{ }^{2}+E_{3}{ }^{2}\right)+\left(\frac{\partial B_{2}}{\partial r}\right)^{2}+\frac{1}{2}\left(\frac{\partial B_{3}}{\partial r}\right)^{2} \\
& +\left(B_{2}-B_{3}\right)^{2}\left(\frac{2}{r^{2}}+\frac{2 C_{1}}{r}\right)+C_{1}{ }^{2}\left(B_{2}{ }^{2}+B_{3}{ }^{2}\right)+W-E_{i} \frac{\partial W}{\partial E_{i}}
\end{aligned}
$$

As we mentioned in Sec. II, we have on physical grounds constrained the parameters in $W$ so that the value of vacuum energy density $\epsilon_{\mathrm{vac}}$ is the same in both the spherical and cylindrical cases. We must, of course, also constrain them so that both cases have eigenvalues with positive real parts for $\alpha$, in order that there are nonsingular asymptotic solutions. Within these constraints there still remains considerable freedom in the choice of parameters. In Table I we list for a number of choices of the numbers characterizing $W$ the values of the mass scales $M$ and $M_{f}$ as well as the glueball mass. In all cases we have chosen the parameters so that the string tension $\kappa$ takes on its "experimental" lattice value of $0.2 \mathrm{GeV}^{2}$, and the vacuum energy density is given by $\epsilon_{\mathrm{vac}}=-0.096 \kappa^{2}$, also as indicated by lattice calculations. ${ }^{12}$ The values $z, x$, and $w$ are the parameters of the cylindrical problem given in I for each case.

We can clearly not make a precise glueball mass prediction; there is still too much freedom in $W$. Nevertheless it is encouraging that there is so little sensitivity in $M_{\text {glueball }}$ to large variations in $\boldsymbol{x}, \boldsymbol{z}$, and $w$, and that a reasonable range of values is obtained. Perhaps further applications of our effective Lagrangian will eventually permit the parameters to be pinned down enough to make specific numerical predictions possible.

\section{PHYSICAL PROPERTIES AND INTERPRETATION OF THE SOLUTION}

The spherical solution we have obtained is one in which all physical quantities are exponentially localized around

TABLE I. Consistent values of glueball masses and the magnetic condensate $G_{2}$ for several of our solutions have a string tension $\kappa$ of $0.2 \mathrm{GeV}^{2}$ and a vacuum energy density $\epsilon_{\mathrm{vac}}=-0.096 \kappa^{2}$. The parameters $\boldsymbol{x}, \boldsymbol{z}$, and $w$ are defined in I.

\begin{tabular}{clllrrrr}
\hline \hline Solution & \multicolumn{1}{c}{$\boldsymbol{z}$} & \multicolumn{1}{c}{$\boldsymbol{x}$} & $\boldsymbol{M}$ & $\begin{array}{c}M \\
(\mathrm{MeV})\end{array}$ & $\begin{array}{c}\boldsymbol{M}_{f} \\
(\mathrm{MeV})\end{array}$ & $\begin{array}{c}\boldsymbol{M}_{\text {glueball }} \\
(\mathrm{GeV})\end{array}$ & $\begin{array}{c}\boldsymbol{G}_{2} \\
\left(\mathrm{GeV}^{4}\right)\end{array}$ \\
\hline$a$ & 0.0107 & -0.155 & 0.0854 & 128 & 621 & 2.44 & 0.0212 \\
$b$ & 0.0213 & -0.310 & 0.1707 & 141 & 400 & 4.60 & 0.0308 \\
$c$ & 0.25 & -1.5 & 1.125 & 153 & 404 & 4.35 & 0.0176 \\
$d$ & 0.5058 & -2.61 & 4.63 & 114 & 480 & 2.00 & 0.0048 \\
$e$ & 0.0170 & -1.833 & 0.094 & 29 & 107 & 2.03 & 0.0004 \\
$f$ & 0.053 & -1.87 & 0.328 & 50 & 187 & 2.07 & 0.0012 \\
$g$ & 2.224 & -1.67 & 34.5 & 221 & 2124 & 1.15 & 0.0095 \\
\hline \hline
\end{tabular}


the center of the excitation. Only the unmeasurable potential $\mathbf{C} \rightarrow-(2 / r) \hat{\mathbf{x}} \times \mathbf{T}$ and the auxiliary field $\mathbf{B} \rightarrow b(\mathbf{T}-2 \widehat{\mathbf{x}} \hat{\mathbf{x}} \cdot \mathbf{T})$ survive far away. The object carries, therefore, no color charge. Indeed, the charges can be derived from the effective Lagrangian. By making the infinitesimal transformation

$$
\begin{aligned}
& C_{\mu} \rightarrow C_{\mu}-i\left[C_{\mu}, \delta \Omega\right], \\
& \widetilde{F}_{\mu v} \rightarrow \widetilde{F}_{\mu v}-i\left[\widetilde{F}_{\mu v}, \delta \Omega\right],
\end{aligned}
$$

the Lagrangian changes to

$$
L_{\text {eff }} \rightarrow L_{\text {eff }}+J_{\mu} \partial_{\mu} \delta \Omega,
$$

where $J_{\mu}$ is the conserved current:

$$
\partial_{\mu} J_{\mu}=0 \text {. }
$$

Carrying out this operation on (1.1) yields the magnetic charge

$$
Q_{M}=\int d^{3} x \nabla \cdot\left(\mathbf{B}+\frac{1}{f^{2}} \mathbf{H}\right)
$$

the electric charge is

$$
Q_{E}=\int d^{3} \mathbf{x} \nabla \cdot\left(\mathbf{E}+\frac{1}{f^{2}} \mathbf{D}\right)
$$

Since both $E$ and $D$ vanish exponentially, the total electric charge of the excitation is zero; it is a color neutral object. On the other hand, as we have seen, the net electric flux crossing any median plane with normal $\hat{\mathbf{n}}$ is $e \hat{\mathbf{n}} \cdot \mathbf{T}$ in the gauge we have used. Thus inside the localized excitation there are a pair of opposite integer charges on opposite sides of the plane. The structure is therefore like two gluons bound closely together. This is evidently a glueball (though, since the color charge density is itself gauge variant, such a description is valid only in this gauge).

The magnetic charge is also zero. Since $\mathbf{H} \rightarrow 0$ and $\mathbf{B} \rightarrow b(\mathbf{T}-2 \widehat{\mathbf{x}} \mathbf{x} \cdot \mathbf{T})$ we have

$$
Q_{M}=b \oint d \mathbf{S} \cdot(\mathbf{T}-2 \widehat{\mathbf{x}} \widehat{\mathbf{x}} \cdot \mathbf{T})
$$

over a surface at infinity, which vanishes.

Inside the glueball, there are rather complicated field configurations. The field lines are shown in Figs. 3(a) and 3(b). $\mathbf{E}, \mathbf{D}$, and $\mathbf{H}$ fall off exponentially at large $r . \mathbf{B}$ is also dipolelike close in, but becomes constant and parallel to the vector $(\mathbf{T}-2 \hat{\mathbf{x}} \hat{\mathbf{x}} \cdot \mathbf{T})$ far out. The vector potential $\mathbf{C}$ wraps around the $\mathbf{T}$ axis in the $\phi$ direction: if $\mathbf{T}$ is the $z$ axis, $\mathbf{C}$ is proportional to $\hat{\mathbf{e}}_{\phi}$.

The mass of the glueball, as we have seen, is not well determined because there is too much numerical freedom in the choice of $W$. All we can say is that it appears to be in the range of one to a few $\mathrm{GeV}$. It is obvious that some
D FIELD LINES IN THE $X-Z$ PLANE

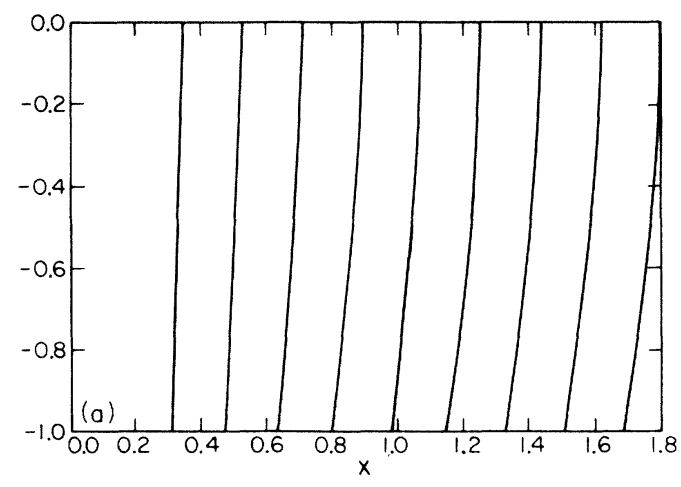

B FIELD LINES IN THE X-Z PLANE

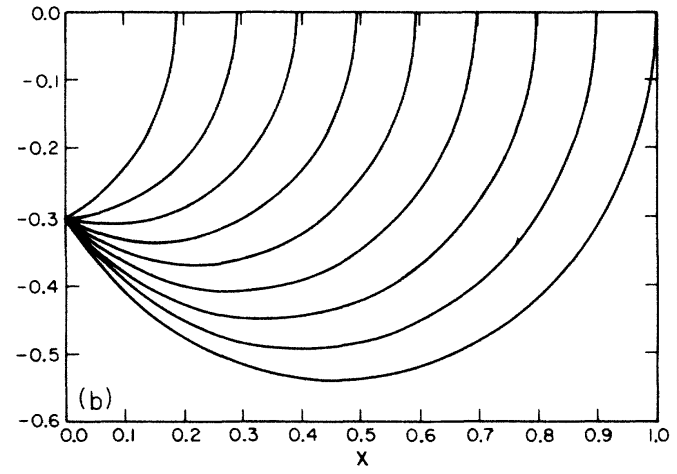

FIG. 3. (a) Field lines of $D$ in the $x-z$ plane. Only one quadrant is shown. (b) Field lines of $\mathbf{B}$ in the $x-z$ plane.

way of simplifying, or further limiting, $W$, would be of great value in constructing a quantitatively useful effective Lagrangian.

One way of doing this would be to choose $W$ in such a way as to allow a smooth interpolation from the infrared, where (1.1) is valid, to the ultraviolet regime, where the effective Lagrangian is also known. What makes this difficult to do is, of course, that (1.1) is known in terms of the dual potential $C_{\mu}$ while in the uv $L_{\text {eff }}$ is known in terms of the ordinary potential $A_{\mu}$, and it is not known how to translate between the two. Nevertheless, we are pursuing approximate ways of implementing this interpolation.

\section{ACKNOWLEDGMENTS}

We would like to thank M. Peskin for a very useful conversation. The work of J.S.B. was supported in part by the National Science Foundation under Grant No. PHY-8405648. The work of F.Z. was supported in part by the U.S. Department of Energy under Contract No. DEAC 03-81-ER40050.
${ }^{1}$ M. Baker, J. S. Ball, and F. Zachariasen, Phys. Rev. D 31, 2575 (1985); hereafter referred to as I.

${ }^{2}$ V. P. Nair and C. Rosenzweig, Phys. Lett. 135B, 450 (1984).

${ }^{3} \mathrm{~F}$. Englert, in Hadron Structure and Lepton-Hadron Interac- tions, Cargese, 1977, edited by M. Lévy et al. (Plenum, New York, 1979), p. 503.

${ }^{4}$ S. Mandelstam, Phys. Rev. D 19, 2391 (1979).

${ }^{5}$ R. P. Feynman and M. Cohen, Phys. Rev. 102, 1189 (1956). 
6M. J. M. Hill, Philos. Trans. R. Soc. London A135, 66 (1894).

${ }^{7}$ M. Baker, J. S. Ball, and F. Zachariasen, Nucl. Phys. B186, 531 (1981).

${ }^{8}$ M. Baker, J. S. Ball, and F. Zachariasen, Nucl. Phys. B226, 455 (1983).
${ }^{9}$ C. DeTar and L. McLerran, Nucl. Phys. B249, 621 (1985).

${ }^{10}$ E. Witten, Phys. Rep. 38, 121 (1977).

${ }^{11} \mathrm{G}$. 't Hooft, Nucl. Phys. B153, 141 (1979).

${ }^{12}$ A. DiGiacomo and G. Paffuti, Phys. Lett. 108B, 327 (1982). 